\title{
电极极化现象的应用
}

\author{
邓玲娟* \\ 咸阳师范学院化学与化工学院, 陕西咸阳 712000
}

摘要: 电极极化是一个非常重要的电化学现象, 但是传统物理化学教材中该部分内容的编排容易让学生对电极极化 现象形成偏见, 认为应当尽量减小或者消除极化现象。本文重点介绍了人们对于电极极化现象的合理应用, 说明极 化现象的存在有利有弊, 合理利用极化现象, 会为我们的生产和生活带来巨大的收益。

关键词: 电极极化; 超电势; 合理应用

中图分类号: G64; O6

\section{Application of the Polarization Effect}

\section{Lingjuan Deng *}

College of Chemistry and Chemical Engineering, Xianyang Normal University, Xianyang 712000, Shaanxi Province, China.

\begin{abstract}
Polarization effect plays an important role in electrochemistry. The content arrangement in the traditional textbooks, however, always makes college students feel that the polarization effect is unreasonable for many aspects, particularly in the field of energy storage and transformation. They often intend to think that it is best to eliminate or reduce the polarization effect. However, just as one coin has two sides, the existence of polarization effect is doubleedged. The reasonable application of polarization effect is introduced in this paper. The examples of application of polarization effect can make college students understand that advantages and disadvantages depend, and reasonable using the polarization effect can bring a significant amount of revenue.
\end{abstract}

Key Words: Polarization effect; Overpotential; Reasonable application

电极极化(polarization)是物理化学课程中电化学部分一个非常重要的不可逆电化学现象, 产生电 极极化的主要原因是浓差极化和电化学极化。由于传统的物理化学教材中过多强调了电极极化以及 由此而产生的超电势在能量利用方面的弊端, 导致学生往往对于电极极化现象存在错误感知: 认为 电极极化以及由此而产生的结果均是不利的因素, 应该着力减弱或消除。本文简单介绍了电极极化 现象, 重点介绍了人们对于电极极化现象的合理利用, 希望对于电极极化及超电势部分教学给出合 理的建议。

\section{1 电极极化}

在电极极化部分, 大部分教材会介绍电极极化产生的原因以及由此而产生的超电势, 并且重点 介绍由于极化现象的存在, 导致原电池的工作电压小于可逆情况下的电池电压(图1a), 而电解池的工 作电压会大于可逆情况下的电池电压(图1b), 这样会造成能源浪费 ${ }^{[1-4]}$, 因此在实际的生产过程中,

收稿: 2021-07-12; 录用: 2021-08-18; 网络发表: 2021-10-25

“通讯作者, Email: denglingjuan@163.com

基金资助: 咸阳师范学院教育教学改革研究项目(2019Y22) 
人们总是要想办法减小或者消除极化现象。而在超电势部分, 一般教材中会详细介绍氢超电势领域 的研究进展, 简略介绍超电势的消除方法。通过这一部分的学习, 学生往往觉得极化现象以及超电 势的存在是非常不利的因素, 因此人们总是要想办法消除或者减小极化现象的发生。
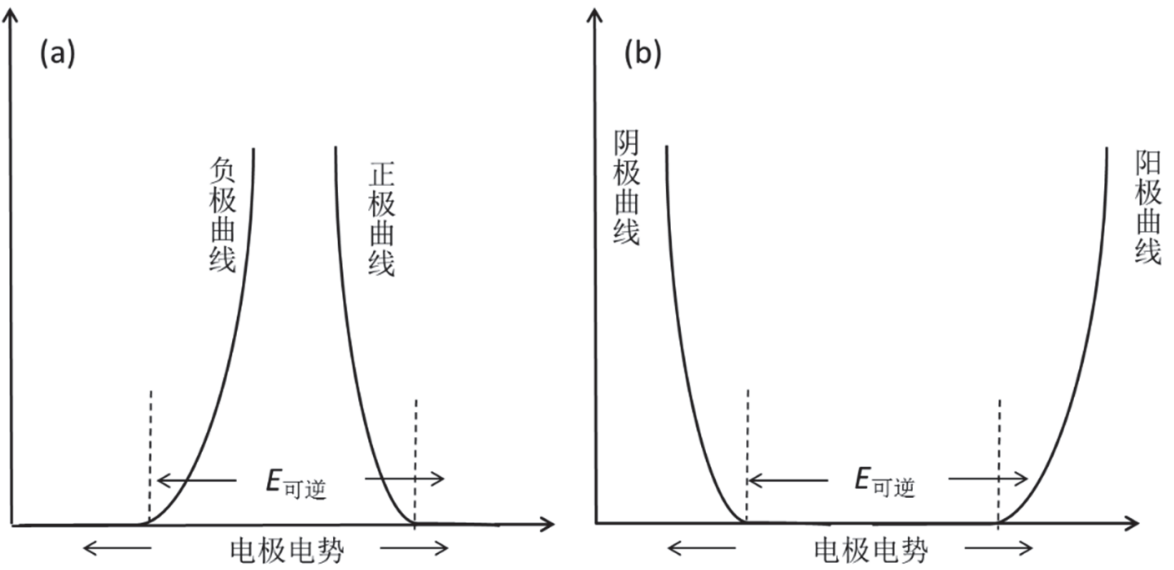

图1 电解池(a)和原电池(b)的极化曲线

实际上, 极化现象是最普通的电化学现象。在电极的金属-电解质界面会产生总厚度约 $0.2-20 \mathrm{~nm}$ 的双电层, 其中由于电极的金属相为电的良导体, 过剩电荷集中在表面; 而由于电解质的电阻较大, 过剩电荷只部分紧贴于相界面, 被称为紧密双层(compact double layer); 余下部分呈分散态, 称分散 双层(diffuse double layer)。由于电极反应的核心步骤都在紧密层中进行, 故双电层结构对于电化学 过程具有重要意义。从双电层的角度考虑, 当电位-电流变化时, 是非法拉第过程, 流向界面的电荷 只用于改变界面构造而不发生电化学反应, 这是理想电容器。但是当电极上发生氧化还原反应时, 有 电荷传递现象发生, 是法拉第过程。因为理想的电容不通过电荷传递, 因此法拉第电极过程破坏了 双电层的理想性, 产生了漏电电流, 这就意味着电极上一旦发生电化学反应, 理想的双电层就破坏 了, 相当于一个漏电的电容器。换而言之, 法拉第电极过程均非理想的可逆过程, 因而电极极化则 是非常普通的电化学现象。

正如那句著名的浐语所述 “每一枚硬币都有正反两面”, 每一个事物的存在都具有两面性, 从能 量利用的角度来看, 极化现象和超电势的存在确实是不利的, 但是其在其他领域却有非常重要的应 用, 下面列举几个人们合理利用电极极化现象及超电势的例子。

\section{2 电极极化现象及超电势的应用}

\section{1 超级电容器}

超级电容 (Supercapacitors) 是一种介于传统电容器 (Capacitor) 和锂离子电容器 (Lithium ion batteries)之间的新型储能器件, 具有功率密度大、循环稳定性好等优势, 已经被广泛用于军事及航空 航天等领域。超级电容器一般由集流体、电极材料和电解液组成(图2a)。目前超级电容器的应用远远 落后于锂离子电池, 原因就是超级电容器的比能量较低。例如, 目前商用碳基超级电容器的比能量 仅为3-5 Wh $\mathrm{kg}^{-1}$, 而常见的锂离子电池的比能量可高达 $100 \mathrm{Wh} \cdot \mathrm{kg}^{-1}$ (图2b)。超级电容器的比能量 $(E$, $\mathrm{Wh} \cdot \mathrm{kg}^{-1}$ ) 计算公式是: $E=1 / 2 C V^{2}$, 其中 $C$ 为电容器的比电容 $\left(\mathrm{F} \cdot \mathrm{g}^{-1}\right)$, 主要由电极材料(碳材料、金 属氧化物和导电聚合物是三种经典的超级电容器电极材料) 的性质决定; 而 $V$ 为电容器的工作电压 $(\mathrm{V})$, 主要由电解质的分解电压来决定。从超级电容器比能量的计算公式可以看出, 在 $C$ 和 $V$ 两个影响 因素中, $V$ 是最主要的因素, 因此要提高超级电容器的能量密度, 最行之有效的方法就是提高电容器 的工作电压。 

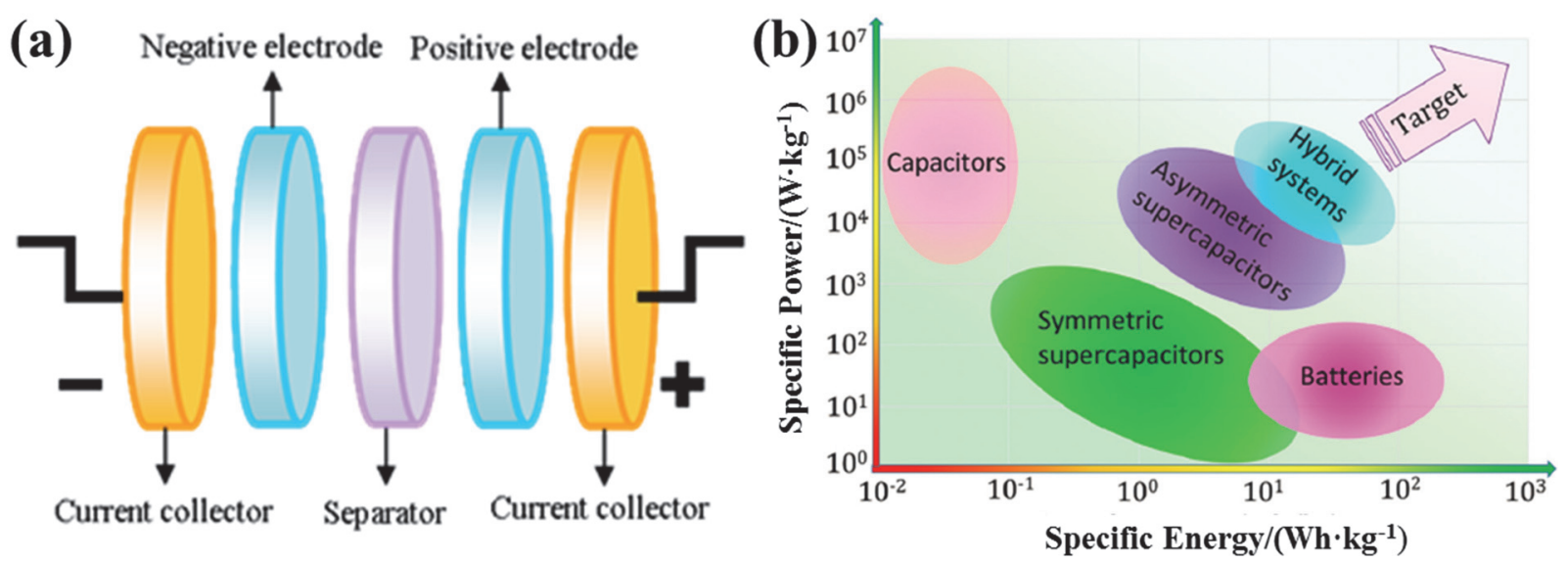

图2 (a) 超级电容器的组装示意图 ${ }^{[5]}$; (b) 传统电容器、超级电容器及电容器的Ragon 曲线 ${ }^{[6]}$

正如前文所述, 电容器的 $V$ 受限于电解液的分解电压, 常见的电解液包括水系和有机体系, 水的 理论分解电压是 $1.23 \mathrm{~V}$, 有机电解质的分解电压可以高达 $4-5 \mathrm{~V}$, 但是基于有机电解质的超级电容器 由于装配工艺复杂, 且需要配备手套箱等昂贵的仪器设备, 因此人们更加倾向于组装基于水体系电 解质的高比能量超级电容器。目前已有很多文献报道基于水系电解质的电容器, 其工作电压高达 $2 \mathrm{~V}$

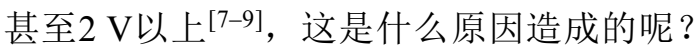

我们课题组曾在 2013 年组装了一个基于碳纳米管/石墨烯/二氧化锰 $\left(\mathrm{CNT} / \mathrm{GR} / \mathrm{MnO}_{2}\right)$ 和碳纳米管/ 石墨烯 $(\mathrm{CNT} / \mathrm{GR})$ 的不对称超级电容器, 电解质为 $1.0 \mathrm{~mol} \cdot \mathrm{L}^{-1}$ 硫酸钠水溶液, 其工作电压为 $2.0 \mathrm{~V}{ }^{[10]}$ 。 基于水性电解质电容器的工作电压受限于以下两个反应:

$$
\begin{aligned}
& 4 \mathrm{OH}^{-} \rightarrow 2 \mathrm{H}_{2} \mathrm{O}+\mathrm{O}_{2}+4 \mathrm{e}^{-} \\
& 4 \mathrm{H}^{+}+4 \mathrm{e}^{-} \rightarrow 2 \mathrm{H}_{2}
\end{aligned}
$$

根据Nernst方程, 在中性电解质中, $\mathrm{O}_{2}$ 和 $\mathrm{H}_{2}$ 的理论析出电势分别为 +0.82 和 $-0.41 \mathrm{~V} v s$. SHE (标准 氢电极)。实验室最常见的参比电极为饱和甘录电极(saturated calomel electrode, SCE)在室温下的电 极电势为 $0.27 \mathrm{~V} v s$. SCE, 因此 $\mathrm{O}_{2}$ 和 $\mathrm{H}_{2}$ 的理论析出电势分别为 +0.55 和 $-0.68 \mathrm{~V} v s$. SCE。图3a给出了 $\mathrm{CNT} / \mathrm{GR}$ 和 $\mathrm{CNT} / \mathrm{GR} / \mathrm{MnO}_{2}$ 电极的循环伏安曲线。从图中可以看出 $\mathrm{CNT} / \mathrm{GR} / \mathrm{MnO}_{2}$ 电极电位窗口为 $0-1.0 \mathrm{~V} v s$. SCE, 而 CNT/GR电极的电位窗口为 $-1.0-0 \mathrm{~V} v s$. $\mathrm{SCE}$ (图3b), 因此CNT/GR/ $/ \mathrm{MnO}_{2}$ 电极的 析氧电势 $(1.0 \mathrm{~V} v s . \mathrm{SCE})$ 高于理论电势, 而 $\mathrm{CNT} / \mathrm{GR}$ 电极的析氢电势 $(-1.0 \mathrm{~V} v s$. SCE) 也低于理论电势,

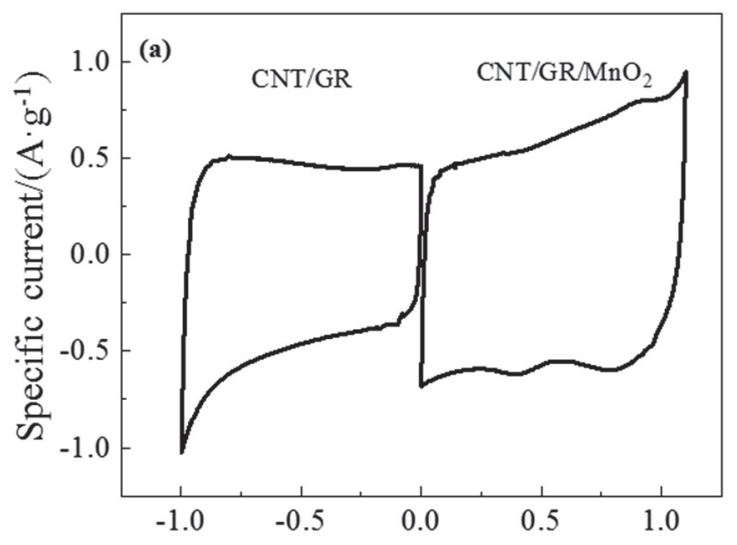

Potential/V vs. SCE

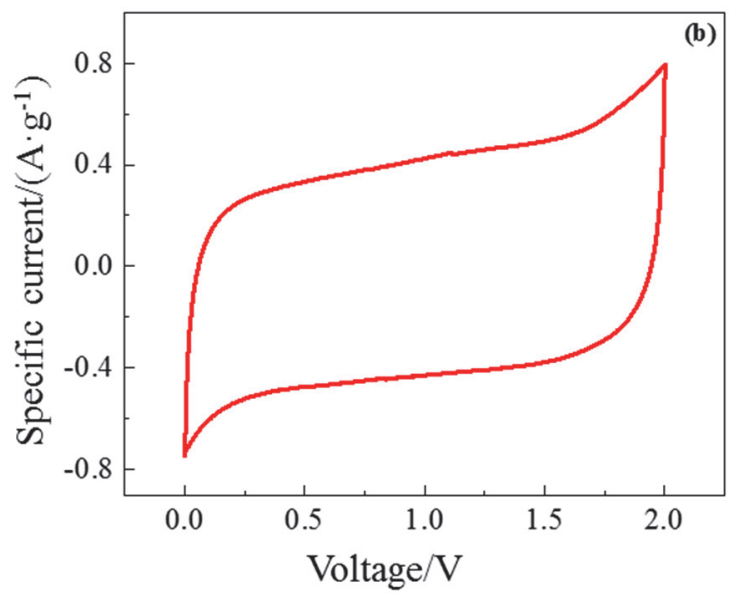

图3 $\mathrm{CNT} / \mathrm{GR} 、 \mathrm{CNT} / \mathrm{GR} / \mathrm{MnO}_{2}(\mathrm{a})$ 及 $\mathrm{CNT} / \mathrm{GR} / \mathrm{MnO}_{2} / / \mathrm{CNT} / \mathrm{GR} / \mathrm{MnO}_{2}$ (b) 电容器在扫速为 $10 \mathrm{mV} \cdot \mathrm{s}^{-1}$ 时的 $\mathrm{CV}$ 曲线 
这些均是由于电极材料在集流体上存在超电势而引起的。

当以 $\mathrm{CNT} / \mathrm{GR} / \mathrm{MnO}_{2}$ 电极为正极, 以 $\mathrm{CNT} / \mathrm{GR}$ 电极为负极, 就可以组装工作电压为 $2.0(1.0-(-1.0)=$ $2.0) \mathrm{V}$ 的超级电容器。当电容器的比电容恒定时, 将电容器的工作电压由 $1.23 \mathrm{~V}$ 增大至 $2.0 \mathrm{~V}$, 可将该 超级电容器的比能量提高至原来的2.6倍。在 $\mathrm{CNT} / \mathrm{GR} / \mathrm{MnO}_{2} / / \mathrm{CNT} / \mathrm{GR} / \mathrm{MnO}_{2}$ 不对称超级电容器中, 极 化现象以及由此而产生的超电势, 均成为了能提升超级电容器比能量的有利因素。

\section{2 铅酸电池}

自1859年法国人普兰特发明铅酸蓄电池以来, 其在交通、通信、电力、军事、航海、航空等各 领域都发挥了举足轻重的作用。铅酸电池的电极主要由铅及其氧化物组成, 电解液是硫酸溶液。铅 酸电池符号可以表示为: $\mathrm{Pb}-\mathrm{PbSO}_{4}\left|\mathrm{H}_{2} \mathrm{SO}_{4}\right| \mathrm{PbO}_{2}-\mathrm{PbSO}_{4}$, 正负极分别对应的电极反应及电极电势为:

$$
\begin{array}{ll}
\mathrm{Pb}-2 \mathrm{e}^{-}+\mathrm{SO}_{4}^{2-} \rightarrow \mathrm{PbSO}_{4} & \varphi_{-}^{\ominus}=-0.3588 \mathrm{~V} \text { vs. SHE } \\
\mathrm{PbO}_{2}+2 \mathrm{e}^{-}+\mathrm{SO}_{4}^{2-}+4 \mathrm{H}^{+} \rightarrow \mathrm{PbSO}_{4}+2 \mathrm{H}_{2} \mathrm{O} & \varphi_{+}^{\ominus}=1.685 \mathrm{~V} \text { vs. SHE }
\end{array}
$$

正如前文所述, 在水系电解质中, 电池的工作电压依然受限于水的分解电压, 因此铅酸电池的 理论工作电压应该为 $1.23 \mathrm{~V}$ 。正是由于极化现象存在, 铅酸电池正极上析氧的电极电势升高, 从而获 得了较高的开路电压。铅酸电池的开路电压为 $2.0 \mathrm{~V}$, 理论比能量为 $166.9 \mathrm{Wh} \cdot \mathrm{kg}^{-1}$ 。在实际应用中, 人们经常将多个单格铅酸电池串联起来以获得电压更大的电池组。

\section{3 电解生产氯气 $\left(\mathrm{Cl}_{2}\right)$}

$\mathrm{Cl}_{2}$ 是一种重要的工业和生活原料, 在工业和生活领域发挥着重要的作用, 目前工业上大规模生 产氯气主要来自于电解饱和食盐水溶液。如图4所示, 该工艺过程具体包括盐水除杂、电解和产品精 制等工序, 其中最主要的工序是电解。工业上电解饱和食盐水的方法包括隔膜电解法、水银电解法 和离子膜电解法, 目前应用较多的是隔膜电解法。

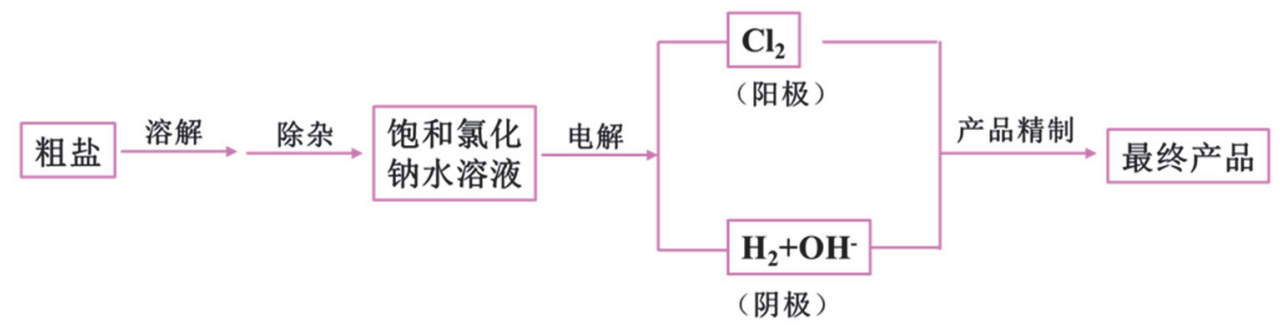

图4 电解法制备氯气流程简图

在电解过程中, 氯化钠水溶液在直流电作用下, 阴离子在阳极上发生氧化反应, 阳离子在阴极 上发生还原反应。电解池的理论分解电压为阳极与阴极的电极电势之差。电解过程中, 在阳极上的 竞争反应有:

$$
\begin{aligned}
& 2 \mathrm{Cl}^{-} \rightarrow \mathrm{Cl}_{2}+2 \mathrm{e}^{-} \\
& 4 \mathrm{OH}^{-} \rightarrow \mathrm{O}_{2}+2 \mathrm{H}_{2} \mathrm{O}+4 \mathrm{e}^{-}
\end{aligned}
$$

析氯反应的可逆电极电势为 $+1.33 \mathrm{~V} v s$. SHE, 该电势会随氯化钠浓度和温度的降低而增大; 析 氧反应的可逆电极电势为 $+0.82 \mathrm{~V} v \mathrm{~s}$. SHE。从分解电压的角度考虑, 析氧反应比析氯反应更容易进 行。但是由于电解池在实际工作时, 流经电解池电流很大, 因此实际电极电势与可逆电极电势并不 相等, 相互竞争的电极反应在电极上实际放电次序取决于实际电极电势, 工业上电解食盐水溶液时 的阳极过程就属于这种情况。

在不同的电极材料表面, 析氧反应和析氯反应的超电势不同。如在生产中常用的钌-钛金属阳极 表面, 当电流密度为 $1000-5000 \mathrm{~A} \cdot \mathrm{m}^{-2}$ 时, 析氧反应的实际电极电势反而比析氯反应的电极电势高 $0.25-0.30 \mathrm{~V}$ 。因此, 在实际电解过程中, 阳极过程主要是析氯而不是析氧。采用提高电解液中氯离 子浓度、控制阳极电解液 $\mathrm{pH}$ 、降低氢氧根离子浓度并采用较高的电流密度等措施, 都可以增大析氧 
和析氯反应的电极电位差, 有利于抑制析氧反应, 从而提高氯气纯度。

在电解饱和食盐水的过程中, 阴极上的竞争反应有:

$$
\begin{aligned}
& \mathrm{Na}^{+}+\mathrm{e}^{-} \rightarrow \mathrm{Na} \\
& 2 \mathrm{H}_{2} \mathrm{O}+2 \mathrm{e}^{-} \rightarrow \mathrm{H}_{2}+2 \mathrm{OH}^{-}
\end{aligned}
$$

由于析钠的电极电势为 $-2.71 \mathrm{~V} v s$. SHE, 因此 $\mathrm{Na}^{+}$很难还原为 $\mathrm{Na}$ 。因而在实际电解过程中, 阴极 上主要发生的反应是水分子被还原为氢气, 并且形成氢氧化钠溶液。

在 $298 \mathrm{~K}$ 时, 电解液中含有氢氧化钠 $\left(100 \mathrm{~g} \cdot \mathrm{L}^{-1}\right)$ 和氯化钠 $\left(180 \mathrm{~g} \cdot \mathrm{L}^{-1}\right)$, 此时析氢的电极电位为 $-0.851 \mathrm{~V} v s$. SHE。因此, $25^{\circ} \mathrm{C}$ 时隔膜电解槽的理论分解电压为:

$$
V_{298 \mathrm{~K}}=\varphi_{\text {阳极 }}-\varphi_{\text {阳极 }}=1.33 \mathrm{~V}-(-0.85 \mathrm{~V})=2.18 \mathrm{~V}
$$

同样由于极化现象和超电势的存在, 使得理论上不可能发生的反应变为现实, 并且为人们利用 来大批量生产 $\mathrm{Cl}_{2}$, 极化现象在此时也变成了一种非常有利的存在。

\section{4 极谱分析}

极谱分析法(polarographic analysis)于1922年由捷克化学家J. Heryovsky建立, 被认为是一种特殊 的电解分析方法，该方法具有灵敏度高、相对误差小、重现性好且检测范围宽等优点 ${ }^{[11]}$ 。

极谱分析法一般采用面积小且易极化的电极作为工作电极(最常采用的工作电极为滴录电极), 选用面积大而不易极化的电极为参比电极 (一般为 SCE), 以含有待测物质的稀溶液为电解质来组成 电解池。在电解过程中, 由于滴永电极表面积很小, 因此电解过程中电流密度很大, 在滴录电极表 面的待测离子很容易得到电子被还原(此时可认为待测离子的浓度趋近于零), 所以就出现了明显的 浓差极化。由于不同的物质有不同的还原电位, 极谱分析法正是根据此原理利用进行定性分析的。 对于同一种物质, 又因为其扩散电流 $\left(i_{\mathrm{d}}\right)$ 与待测物质浓度 $(c)$ 呈线性关系: $i_{\mathrm{d}}=k c$, 这又为利用极谱法 进行定量分析带来了可能。由于在电解过程中, 滴录电极的表面可以不断更新, 该测试方法又具有 重现性好的优点。

虽然随着人们对于环境问题的日益关注, 考虑到录具有毒性, 极谱分析法已经逐渐被淘汰, 但 是电极极化也曾经为人们利用, 发展了一种灵敏的电化学分析方法。

除了以上提到的几个方面, 极化现象及超电势在电镀工程、金属防腐、电化学氧化处理废水 ${ }^{[12] 、}$ 及电解铜等领域 ${ }^{[13]}$ 均具有重要的应用价值。

\section{3 结语}

电极极化现象是一个常见的电化学现象, 而传统物理化学教材中的导向会给学生对于其理解带 来误区, 如果教师不加以引导, 学生往往会形成一定的思维定式。如果教师在授课过程中对学生加 以适当的引导, 让学生自己查阅文献, 去探究事实的真相, 这样既锻炼了学生理性探究问题的能力, 培养学生辩证思维的能力, 也会对电极极化以及由于电极极化而产生的结果有一个更加全面和理性 的认识。

\section{参 考 文 献}

[1] 傅献彩, 沈文霞, 姚天扬, 侯文华. 物理化学. 北京: 高等教育出版社, 2015: 119-129.

[2] 李松林, 周亚平, 刘俊吉. 物理化学. 北京: 高等教育出版社, 2015: 350-352.

[3] 沈文霞. 物理化学核心教程. 北京: 科学出版社, 2009: 305-309.

[4] 印永嘉, 奚正楷, 张树永. 物理化学简明教程. 北京: 高等教育出版社, 2007: 350-352.

[5] 邓玲娟. 过渡金属氧化物/石墨烯纳米电极材料制备及其电容性质[博士学位论文]. 西安: 陕西师范大学, 2013.

[6] Noori, A.; El-Kady, M. F.; Rahmanifar, M. S.; Kaner, R. B.; Mousav. M. F. Chem. Soc. Rev. 2019, 48, 1272.

[7] Wang, J.-G.; Ren, L.; Hou, Z.; Shao, M. Chem. Eng. J. 2020, 397, 125521. 
[8] Pan, Q.; Yang, C.; Jia, Q.; Qi, W.; Wei, H.; Wang, M.; Yang, S.; Cao, B. Chem. Eng. J. 2020, 397, 125524.

[9] Dang, S.; Wen, Y.; Qin, T.; Hao, J.; Li, H.; Huang, J.; Yan, D.; Cao, G.; Peng, S. Chem. Eng. J. 2020, $396,125342$.

[10] Deng, L.; Hao, Z.; Wang, J.; Zhu, G.; Kang, L.; Liu, Z.-H. Electrochim. Acta 2013, 89, 191.

[11] 朱文滨, 刘茵. 净水技术, 2016, 35 (1), 91 .

[12] 万亚珍, 张文辉. 环境科学与技术, 2006, 299 (5), 109.

[13] 唐文忠, 王玉芳. 湖南有色金属, 2007, 23 (1), 13. 